\title{
Clinical and Molecular Characterisation of Children with Pierre Robin Sequence and Additional Anomalies
}

\author{
Jessie X. Xu $\mathrm{u}^{\mathrm{a}, \mathrm{b}}$ Nicky Kilpatrick ${ }^{\mathrm{a}-\mathrm{c}}$ Naomi L. Baker ${ }^{\mathrm{a}} \quad$ Anthony Penington $^{\mathrm{a}-\mathrm{c}}$ \\ Peter G. Farlie ${ }^{a, b}$ Tiong Yang Tan ${ }^{a-d}$ \\ ${ }^{a}$ Murdoch Childrens Research Institute, ${ }^{b}$ Department of Paediatrics, University of Melbourne, 'Royal Children's \\ Hospital, and ${ }^{d}$ Victorian Clinical Genetics Services, Melbourne, Vic., Australia
}

\section{Key Words}

Birth defects · Craniofacial anomalies · Dysmorphology ·

Noncoding DNA · Phenotyping · SOX9

\begin{abstract}
Pierre Robin Sequence (PRS) is usually classified into syndromic and nonsyndromic groups, with a further subclassification of the nonsyndromic group into isolated PRS and PRS with additional anomalies (PRS-Plus). The aim of this research is to provide an accurate phenotypic characterisation of nonsyndromic PRS, specifically the PRS-Plus subgroup. We sought to examine the frequency of sequence variants in previously defined conserved noncoding elements (CNEs) in the putative enhancer region upstream of SOX9, the regulation of which has been associated with PRS phenotypes. We identified 141 children with nonsyndromic PRS at the Royal Children's Hospital, Melbourne from 1985 to 2012 using 2 databases. Clinical and demographic data were extracted by file review and children categorized as 'isolated PRS' or 'PRSPlus'. A subset of children with PRS-Plus was selected for detailed phenotyping and DNA sequencing of the upstream SOX9 CNEs. We found 83 children with isolated PRS and 58 with PRS-Plus. The most common PRS-Plus malformations in-
\end{abstract}

volved the musculoskeletal and ocular systems. The most common coexisting craniofacial malformation was choanal stenosis/atresia. We identified 10 children with a family history of PRS or cleft palate. We found a single nucleotide substitution in a putative GATA1-binding site in one patient, but it was inherited from his phenotypically unaffected mother. PRS-Plus represents a broad phenotypic spectrum with uncertain pathogenesis. Dysmorphology assessment by a clinical geneticist is recommended. SOX9 CNE sequence variants are rare in our cohort and are unlikely to play a significant role in the pathogenesis of PRS-Plus.

ㄷ) 2016 S. Karger AG, Basel

The clinical features of Pierre Robin Sequence (PRS; OMIM 261800) include micrognathia, glossoptosis, and upper airway obstruction [Robin, 1923]. Cleft palate, a variable feature, has often been incorporated into the definition. PRS is most commonly described in terms of 2 broad categories: syndromic and nonsyndromic. Syndromic PRS refers to children with the traditional features as a component of a known syndrome, of which there are more than 50 [summarized in Tan et al., 2013]. Nonsyndromic PRS can be further subcategorized into

\section{KARGER}

E-Mail karger@karger.com

www.karger.com/msy
(C) 2016 S. Karger AG, Basel

$1661-8769 / 16 / 0076-0322 \$ 39.50 / 0$
Tiong Yang Tan

Victorian Clinical Genetics Services

4th Floor Royal Children's Hospital

Flemington Road, Parkville, VIC 3052 (Australia)

E-Mail tiong.tan@vcgs.org.au 
children with: only the core components (isolated PRS) or additional malformations, but not in a pattern recognized as a known syndrome or genetic condition (which we hereafter term PRS-Plus). PRS is clearly a clinically heterogeneous condition, and the full phenotypic spectrum of the nonsyndromic PRS group requires detailed characterisation.

Several theories have been proposed to explain the pathogenesis of PRS, but the most prevailing involves the notion of primary mandibular hypoplasia. This theory states that during embryonic development, an intrinsic or extrinsic factor leads to micrognathia, which in turn causes failure of the tongue to drop from between the palatal shelves resulting in cleft palate [Cohen, 1999].

The identification of microdeletions, translocation breakpoints, and sequence variants disrupting putative regulatory elements in the chromosome $17 \mathrm{q} 24$ noncoding regions far up- and downstream of SOX9 (OMIM 608160 ) in children with isolated PRS provided the first definitive insight into the pathogenesis of PRS [Benko et al., 2009]. Disruption of SOX9 by intragenic mutations and more proximal chromosomal translocation breakpoints cause campomelic dysplasia (OMIM 114290), characterized by skeletal dysplasia, genital anomalies including sex reversal, and craniofacial involvement with PRS as a component feature. Additional support for the dysregulation of SOX9 in the pathogenesis of PRS has been provided by the identification of chromosomal anomalies in children with PRS-Plus, notably involving the skeletal system [Fukami et al., 2012; Gordon et al., 2014; Smyk et al., 2015].

The optimal criteria for diagnosis and management of children with PRS remain contentious. There is no consensus about a definition of PRS and optimal management of the airway and feeding difficulties. Without a comprehensive understanding of the full phenotypic spectrum of syndromic and nonsyndromic PRS, accurate diagnoses and tailored management strategies are challenging and difficult to evaluate systematically. The aim of this research is to provide a detailed phenotypic description of a cohort of children with PRS managed at one tertiary children's hospital, with a specific focus on the PRS-Plus group. A secondary objective of this study was to identify sequence variants in previously described [Gordon et al., 2014] conserved noncoding elements (CNEs) in the 'PRS region' $\sim 1.2 \mathrm{Mb}$ upstream of SOX9 in children with PRS and musculoskeletal anomalies and/or a family history of cleft or PRS as we hypothesized that this group would be most likely to have an underlying genetic basis.

Detailed Characterisation of PRS-Plus

\section{Methods}

Data Collection and Phenotypic Classification

Our cohort of children with nonsyndromic PRS was identified from the Royal Children's Hospital (RCH) in Melbourne, the major tertiary referral centre for PRS in the state of Victoria, Australia. The RCH Cleft Registry and the Victorian Clinical Genetics Services (VCGS) database were cross-referenced to identify all children with PRS born between January 1985 and December 2012. Clinical and demographic data were extracted by detailed review of the RCH medical record and VCGS genetic file. Any children with syndromic PRS (established by clinical or molecular means) were excluded from the study.

We defined isolated PRS as referring to children with only the traditional features of PRS, and PRS-Plus was defined as children with the core features plus one or more congenital anomaly. Trivial or clinically insignificant cardiac lesions (e.g., spontaneously closing patent ductus arteriosus) and common medical conditions such as asthma, eczema, gastroesophageal reflux, and conductive hearing loss secondary to middle ear effusion were not considered significant enough to constitute a diagnosis of PRS-Plus.

\section{Subgroup Selection}

We selected patients with a family history of PRS or cleft from the nonsyndromic PRS cohort (isolated PRS and PRS-Plus) for clinical review and molecular characterisation. Based on the clinical and molecular link with campomelic dysplasia, we also selected a subgroup of PRS-Plus children with musculoskeletal anomalies for the same analysis.

\section{Clinical Assessment and Molecular Characterisation}

Dysmorphology and anthropometric data were collected by clinical assessment using a standardized form (J.X.X.) with independent verification (T.Y.T.). DNA from lymphocytes in saliva was extracted by standard manufacturer protocol (Oragene, Ottawa, Canada). Standard Sanger sequencing of 14 CNEs described previously [Gordon et al., 2009] was performed. Mutation Surveyor software version 3.2 (by Soft Genetics) was used to align sequencing data with the reference genome on the UCSC (University of California, Santa Cruz, Calif., USA) Genome Browser (http://genome.ucsc.edu/), version number GRCh37/hg19 and SNP database version number dbSNP141. CNE variants were examined in the UCSC Genome Browser and primary databases including Transfac (http://www.gene-regulation.com/pub/databases.html) and 1000 Genomes (1000genomes.org) [Matys et al., 2006; 1000 Genomes Project Consortium et al., 2015].

\section{Results}

We identified 174 children with PRS in the RCH Cleft Registry and VCGS database. After 33 children were excluded for having a diagnosed syndrome or pathogenic chromosomal abnormality (table 1), 141 children remained as the nonsyndromic PRS cohort. All children in our cohort had cleft palate as they were ascertained through the cleft registry. Note that the 2 related children 


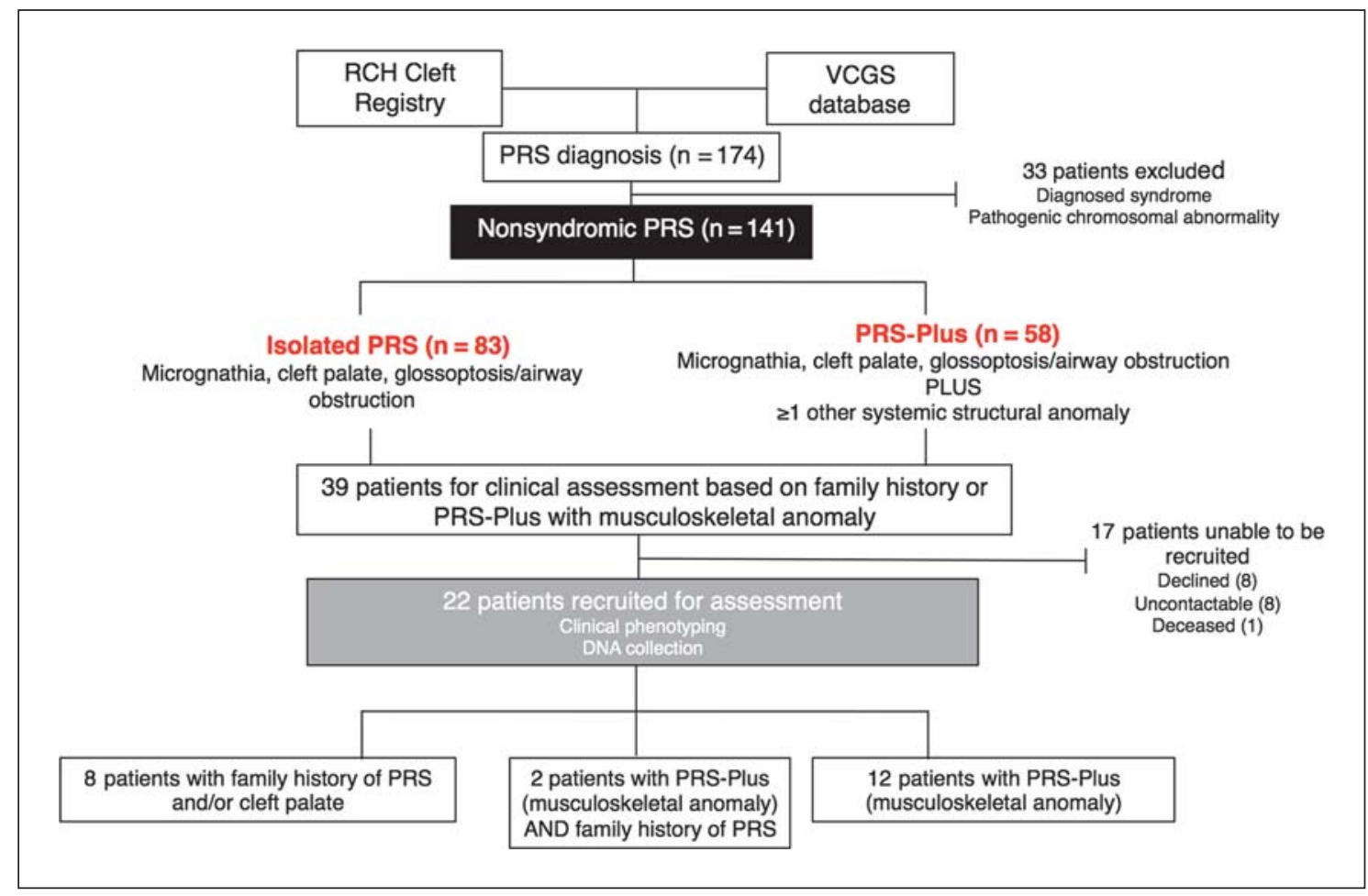

Fig. 1. Methods. This flowchart details the process of the medical record review of the PRS cohort followed by subgroup selection for detailed phenotyping and molecular analysis.

Table 1. Diagnoses of syndromic PRS excluded from study

\begin{tabular}{ll}
\hline Reason for exclusion & $\begin{array}{c}\text { Patients } \\
(\mathrm{n}=33)\end{array}$ \\
\hline Stickler syndrome & 11 \\
22q11 deletion syndrome & 2 \\
22q12 deletion syndrome & 2 \\
Translocation 2;17 & 2 \\
Marshall syndrome & 1 \\
Foetal alcohol syndrome & 1 \\
Treacher-Collins & 1 \\
Deletion 5q & 1 \\
Duplication 7q & 1 \\
Duplication 4q35 & 1 \\
Deletion 19q13.43 & 1 \\
Duplication 8p23.3 & 1 \\
Van der Woude syndrome & 1 \\
Rapp-Hodgkin syndrome & 1 \\
Fragile X syndrome & 1 \\
Smith Magenis syndrome & 1 \\
Campomelic dysplasia & 1 \\
Mandibulofacial dysostosis & 1 \\
Translocation 9;11 & 1 \\
Osteopathia striata with cranial sclerosis & 1 \\
\hline
\end{tabular}

with a balanced $t(2 ; 17)$ translocation have been previously reported [Jamshidi et al., 2004]. Of the 141 children with nonsyndromic PRS, 83 were classified as having isolated PRS and 58 with PRS-Plus by medical record review (fig. 1). Demographic information of the nonsyndromic PRS (isolated PRS and PRS-Plus) cohort is detailed in table 2 .

\section{Phenotypes of Nonsyndromic PRS}

Of the 58 children with PRS-Plus, 36 (62\%) had additional craniofacial dysmorphology noted from medical record review (online suppl. table 1; for all suppl. material, see www.karger.com/doi/10.1159/000449115). The most common facial dysmorphic features were hypertelorism (6), low-set ears (5), and preauricular skin tags (4). Musculoskeletal and ocular anomalies were the most frequently observed abnormalities, with $26 / 58$ (44.8\%) and 23/58 (39.6\%) children affected, respectively. Of the 26 children with musculoskeletal anomalies, the most common conditions were congenital talipes equinovarus $(n=4)$, congenital dislocated hips $(\mathrm{n}=4)$, metatarsus varus $(\mathrm{n}=3)$, pectus excavatum $(\mathrm{n}=2)$, and scoliosis $(\mathrm{n}=2)$. Strabismus was the most common ocular anomaly $(n=9)$. 
Thirty-four of the 58 children with PRS-Plus (59\%) had malformations affecting only one body system beyond the craniofacial region (online suppl. table 2). Two or more body systems beyond the craniofacial region were affected in 24 children with PRS-Plus (41\%). The central nervous system, whilst uncommonly affected in single-system children, was the third most common body system involved in children with multisystem anomalies. These included epilepsy $(n=3)$, hypoplasia of the corpus callosum $(n=2)$, sensorineural hearing loss $(n=2)$, and cerebral palsy $(\mathrm{n}=2)$. All anomalies found in each of the 24 multisystem-affected children are listed in online supplementary table 3 .

The most common craniofacial anomaly besides PRS in our cohort was choanal stenosis/atresia, with 8 children having this condition; 3 as the only additional malformation beyond PRS, and 5 had other associated anomalies.

\section{Family History of Cleft}

Of the 136 children in our nonsyndromic PRS cohort with documented family history, $22(16.2 \%)$ had a relative with orofacial cleft; of these, $13(59.1 \%)$ occurred in a first-degree relative, and 9 (40.9\%) occurred in a seconddegree or more distant relative. These were diagnosed with PRS $(n=9 ; 40.9 \%)$ or cleft palate only $(n=6 ; 27.3 \%)$. There was one pair of siblings with isolated PRS, and their father had cleft palate only.

\section{Detailed Phenotyping of the Selected Subgroup}

On the basis of family history of PRS or cleft, or PRSPlus with musculoskeletal involvement, 39 children were selected for comprehensive phenotyping, but 17 of these children were unable to be recruited for various reasons (fig. 1). Of the remaining 22 with a musculoskeletal anomaly (12), family history of cleft/PRS (8), or both (2), we identified additional dysmorphic features in 7 (31.8\%) children, but did not make any syndrome diagnosis, nor did their PRS classification change. Approximately 60\% of this selected subset (13/22) had already seen a clinical geneticist as part of standard care.

DNA Sequencing of the Noncoding Elements of SOX9

Sanger sequencing of 14 CNEs [Gordon et al., 2009] in the upstream region of SOX9 was undertaken in 22 children with PRS and either a musculoskeletal anomaly (12) or a family history of cleft/PRS (8), or both (2). In total, 4 children $(4 / 22 ; 18.2 \%)$ were found to have a SOX9 CNE variant observed in dbSNP141 at an allele frequency of less than $1 \%$ (online suppl. table 4 ). These 4 children com-
Table 2. Demographic characteristics of the entire nonsyndromic PRS cohort $(\mathrm{n}=141)$, with subclassification into isolated PRS ( $\mathrm{n}=$ $83)$ and PRS-Plus $(n=58)$ groups

\begin{tabular}{lclr}
\hline & Total cohort & Isolated PRS & PRS-Plus \\
\hline Current age, years & & & \\
$1-5$ & $33(23.4)$ & $21(25.3)$ & $12(20.7)$ \\
$6-10$ & $33(23.4)$ & $25(30.9)$ & $8(13.8)$ \\
$11-15$ & $41(29.1)$ & $20(24.1)$ & $21(36.2)$ \\
$16-20$ & $22(15.6)$ & $11(13.3)$ & $11(19.0)$ \\
$21+$ & $12(8.5)$ & $6(7.2)$ & $6(103)$ \\
Gender & & & \\
Male & $62(44.0)$ & $30(36.1)$ & $32(55.1)$ \\
Female & $79(56.0)$ & $53(63.9)$ & $26(44.8)$ \\
Mortality & & & \\
Alive & $139(98.6)$ & $82(98.8)$ & $57(98.3)$ \\
Deceased & $2(1.4)$ & $1(1.2)$ & $1(1.7)$ \\
\hline
\end{tabular}

Percentages are given in parentheses.

prised 3 with PRS-Plus and musculoskeletal anomalies, while 1 had isolated PRS with a family history of cleft palate (online suppl. table 5). In the 3 children with PRS-Plus and musculoskeletal anomalies, heterozygous variants were identified within CNEs 1, 3, and 4. Of interest was the variant occurring in $\mathrm{CNE} 1$ within a predicted GATA1 transcription factor binding site. This was found in a patient (PRS136) with PRS, talipes valgus, and mild pectus excavatum. The single heterozygous nucleotide substitution ( $\mathrm{T}$ to $\mathrm{C}$ ) was identified in CNE1 in chr17:68658181 (USCS browser hg19 build) located $1.459 \mathrm{Mb}$ from the start codon of SOX9. The change was present in 1 of 2,298 samples reported in the dbSNP141 database, but not in 2,504 samples in 1000 Genomes. Parental analysis confirmed that the variant was inherited from a phenotypically unaffected mother.

\section{Discussion}

\section{Phenotypic Spectrum of Children with Nonsyndromic} PRS

The nomenclature, and more importantly, categorisation of children with PRS and additional anomalies without a defined syndrome have traditionally been inconsistent. Some studies refer to this group as PRS with associated anomalies [Holder-Espinasse et al., 2001; Bütow et al., 2009; Caouette-Laberge et al., 2012; Thouvenin et al., 2013], whilst others prefer Unique-PRS [Smith and Senders, 2006] (online suppl. table 6). These children are 
sometimes grouped with syndromic PRS [Shprintzen, 1988, 1992; Marques et al., 1998; Cruz et al., 1999; van den Elzen et al., 2001; Li et al., 2002; Printzlau and Andersen, 2004; Evans et al., 2006; de Buys Roessingh et al., 2007; Izumi et al., 2012; Patel et al., 2012; Gomez-Ospina and Bernstein, 2016] but also grouped with nonsyndromic children in other studies [Sheffield et al., 1987; Bütow et al., 2009; Al-Samkari et al., 2010]. These differences in classification of the PRS-Plus group make interstudy comparisons challenging. Our study sought to develop a more detailed phenotypic analysis of those in the PRSPlus group, representing $41 \%$ of our cohort. These children appear at this time not to be able to be diagnosed with any known syndrome, even after assessment by a clinical geneticist. Since 2010, it has been standard practice at our centre to refer all neonates with PRS for clinical genetics assessment, and an ophthalmology assessment, especially in children with myopia, to exclude Stickler syndrome.

Positional limb deformities are one of the most commonly observed additional anomalies in children with PRS [Smith, 1961; Hanson and Smith, 1975; Williams et al., 1981; Caouette-Laberge et al., 1994; Bütow et al., 2009]. Extrinsic forces such as multiple pregnancy and oligohydramnios have been hypothesized to have an impact on the in utero positioning of the jaw leading to micrognathia [Poswillo, 1966; DeMyer and Baird, 1969; Knottnerus et al., 2001; Aggarwal and Kumar, 2003]. Positional limb deformities such as congenital talipes equinovarus and metatarsus varus may also occur as a result of compressive forces in utero, such as polyhydramnios, oligohydramnios, or twin pregnancy. All the PRS-Plus children with positional limb deformities in this cohort $(\mathrm{n}=$ 7) were singleton pregnancies. Two pregnancies were complicated by polyhydramnios, one pregnancy was complicated by intrauterine growth restriction, and another by premature rupture of membranes at 20 weeks' gestation with resultant oligohydramnios. Of these 4 children with concomitant pregnancy complications, 3 were categorized as PRS-Plus with multisystem anomalies. Our observation that the positional limb deformity occurred with other systemic anomalies suggests an aetiology that is more complex than amniotic fluid disturbance in these children.

\section{Noncoding Elements of SOX9}

Our focus on the CNEs upstream of SOX9 was largely driven by previous work implicating their involvement in the pathogenesis of the nonsyndromic PRS phenotype [Benko et al., 2009; Fukami et al., 2012; Amarillo et al.,
2013; Gordon et al., 2014; Smyk et al., 2015]. Given the clinical and molecular link with campomelic dysplasia (OMIM 114290), we hypothesized that children with PRS and additional skeletal anomalies might have CNE sequence variants that alter $\mathrm{SOX} 9$ expression and impact on skeletal development. We also reasoned that those with a family history of PRS or cleft would be more likely to have a genetic basis than those without.

Genetic analysis in this study was restricted to identifying sequence variants in CNEs that might act as putative regulatory elements of $S O X 9$. The majority $(13 / 22 ; 59.1 \%)$ of the children in the subgroup analysis had seen a clinical geneticist and had been investigated with either a chromosome microarray or conventional karyotyping with FISH for 22q11 deletion. Only one child had a microarray abnormality (maternally inherited 153-kb duplication of chromosome $5 \mathrm{q} 35.5$ ) that was not considered causative for his phenotype.

In one patient with PRS-Plus (talipes valgus and pectus excavatum in addition to PRS), we identified a variant in a predicted GATA1 transcription factor binding site, located within CNE1 upstream of SOX9 (genome location chr17:68658181). The same variant had been identified in 1 out of 2,298 samples reported in the dbSNP141 database. There are no publically available phenotypic data for this single database individual. GATA1 (OMIM 305371) is located in the $\mathrm{X}$ chromosome and codes for a protein which plays an important role in erythroid development [Simon et al., 1992], but no known regulatory interaction with SOX9. Germline mutations in GATA1 cause various hematological diseases [Nichols et al., 2000; Mehaffey et al., 2001; Yu et al., 2002; Hollanda et al., 2006; Sankaran et al., 2012], but are not known to be associated with PRS. Our finding that this variant was inherited from a phenotypically unaffected mother suggests that it is either a benign change or a predispositional variant with incomplete penetrance but unlikely to be the sole pathogenic factor. The other CNE variants identified in 3 other patients ( 2 with PRS-Plus; 1 with family history of cleft) were reported with higher frequency and did not occur within any known transcription factor binding sites, thus making their role in pathogenesis less likely.

CNE sequencing in our cohort of PRS-Plus children with musculoskeletal anomalies and family history of PRS/cleft did not identify any likely causal variants that might impact upon SOX9 expression, suggesting that these are unlikely in this cohort. The significance of the variant overlying the GATA1 transcription factor binding site is unclear, although functional studies beyond the scope of this manuscript may provide clarity. Chromo- 
some microarray has been used to detect large chromosomal deletions $5^{\prime}$ of SOX9 in individuals with PRS [Fukami et al., 2012; Amarillo et al., 2013; Smyk et al., 2015; Castori et al., 2016]. Although chromosome microarray is the genetic investigation of choice in all children with PRS seen after 2010 in our centre, probe coverage is typically low in the agenic $5^{\prime}$ region of $S O X 9$, so it is possible that small copy number variants in this region have been missed in our cohort. Furthermore, our sequencing analysis is unable to detect small copy number variations at our locus of interest. Customized targeted analysis with MLPA of noncoding agenic regions [White et al., 2011] may be more likely to detect such molecular lesions as a cause for PRS-Plus.

\section{Conclusion}

A broad spectrum of phenotypes exists for children with nonsyndromic PRS. Many of these children have anomalies outside of the craniofacial system (PRS-Plus), with musculoskeletal and ocular anomalies being the most common. The PRS-Plus group is fascinating, and it remains unclear as to what genetic factors exist that differentiate it from isolated PRS. This, and all previous work examining the clinical features of PRS, underscores the broad phenotypic spectrum ranging from simple micrognathia to complex syndromes.

The phenotypic spectrum in children with PRS as well as the growing number of syndromes with PRS as a component feature suggests that review by a clinical geneticist is desirable. Our analysis of the CNEs of SOX9 in a selected group of children with musculoskeletal anomalies and/or a family history of cleft or PRS did not reveal any significant mutations in this genomic region, suggesting that these are likely to be rare at least in this subset of PRS. A more agnostic whole genome approach is likely to be of higher yield than a targeted analysis. The breadth of the phenotypic spectrum observed in the PRS-Plus group is difficult to reconcile with a single embryological aetiology. Genetic heterogeneity in PRS has been proposed previously and is a broadly accepted concept. The recent explosion in genetic diagnoses following exome sequencing has highlighted the requirement for detailed phenotyping in gene discovery. However, isolated PRS is by definition a highly constrained phenotype, making dissection of the isolated PRS group to clarify genetic heterogeneity problematic. In contrast, the breadth of the PRS-Plus phenotypes provides an opportunity to define subcategories of PRS based on detailed phenotyping. Once defined, these subgroups will be amenable to contemporary exomebased gene identification strategies, which in turn will provide candidates for examination in isolated PRS cohorts. Further studies are required to fully elucidate the genetic mechanisms underlying the nonsyndromic PRS phenotype, but systematic subphenotyping may provide the most efficient path to this end.

\section{Acknowledgements}

We are grateful to the children and families who participated in this research. We also thank Sue Poynton for assistance with the $\mathrm{RCH}$ cleft registry, David Blake for assistance with the VCGS database, and Supriya Raj for assistance with patient recruitment and ethics submissions. The Victorian Government's Operational Infrastructure Support Program supported this work. The Australian National Health and Medical Research Council Overseas Postdoctoral Training Scholarship (\#607431) supported T.Y.T, and J.X.X. was supported by a University of Melbourne scholarship and submitted this work in fulfilment of the requirements for the degree of Master of Surgery (University of Melbourne).

\section{Statement of Ethics}

Approval for this study was granted by The Royal Children's Hospital Human Research Ethics Committee (approval numbers 32268A and 31190B and C).

\section{Disclosure Statement}

The authors have no conflicts of interest to declare. The authors have not received any grant, honorarium or other form of payment to produce the manuscript.

Aggarwal S, Kumar A: Fetal hydrocolpos leading
to Pierre Robin sequence: an unreported ef-
fect of oligohydramnios sequence. J Perinatol
23:76-78 (2003).
Al-Samkari HT, Kane AA, Molter DW, Vach-
harajani A: Neonatal outcomes of Pierre Rob-
in sequence: an institutional experience. Clin
Pediatr (Phila) 49:1117-1122 (2010).
Amarillo IE, Dipple KM, Quintero-Rivera F: Fa-
milial microdeletion of 17q24.3 upstream of
SOX9 is associated with isolated Pierre Robin
sequence due to position effect. Am J Med
Genet A 161A:1167-1172 (2013).
Benko S, Fantes JA, Amiel J, Kleinjan DJ, Thomas
S, et al: Highly conserved non-coding ele-
ments on either side of SOX9 associated with
Pierre Robin sequence. Nat Genet 41:359-364
(2009).


Bütow KW, Hoogendijk CF, Zwahlen RA: Pierre Robin sequence: appearances and 25 years of experience with an innovative treatment protocol. J Pediatr Surg 44:2112-2118 (2009).

-Caouette-Laberge L, Bayet B, Larocque Y: The Pierre Robin sequence: review of 125 cases and evolution of treatment modalities. Plast Reconstr Surg 93:934-942 (1994).

-Caouette-Laberge L, Borsuk DE, Bortoluzzi PA: Subperiosteal release of the floor of the mouth to correct airway obstruction in pierre robin sequence: review of 31 cases. Cleft Palate Craniofac J 49:14-20 (2012).

Castori M, Bottillo I, Morlino S, Barone C, Cascone $\mathrm{P}$, et al: Variability in a three-generation family with Pierre Robin sequence, acampomelic campomelic dysplasia, and intellectual disability due to a novel $\sim 1 \mathrm{Mb}$ deletion upstream of SOX9, and including KCNJ2 and KCNJ16. Birth Defects Res A Clin Mol Teratol 106:61-68 (2016).

Cohen MM Jr: Robin sequences and complexes: causal heterogeneity and pathogenetic/phenotypic variability. Am J Med Genet 84:311315 (1999).

DCruz MJ, Kerschner JE, Beste DJ, Conley SF: Pierre Robin sequences: secondary respiratory difficulties and intrinsic feeding abnormalities. Laryngoscope 109:1632-1636 (1999).

de Buys Roessingh AS, Herzog G, Hohlfeld J: Respiratory distress in Pierre Robin: successful use of pharyngeal tube. J Pediatr Surg 42: 1495-1499 (2007).

DeMyer W, Baird I: Mortality and skeletal malformations from amniocentesis and oligohydramnios in rats: cleft palate, clubfoot, microstomia, and adactyly. Teratology 2:33-37 (1969).

- Evans AK, Rahbar R, Rogers GF, Mulliken JB, Volk MS: Robin sequence: a retrospective review of 115 patients. Int J Pediatr Otorhinolaryngol 70:973-980 (2006).

-Fukami M, Tsuchiya T, Takada S, Kanbara A, Asahara $\mathrm{H}$, et al: Complex genomic rearrangement in the SOX9 $5^{\prime}$ region in a patient with Pierre Robin sequence and hypoplastic left scapula. Am J Med Genet A 158A:15291534 (2012).

1000 Genomes Project Consortium, Auton A, Brooks LD, Durbin RM, Garrison EP, et al: A global reference for human genetic variation. Nature 526:68-74 (2015).

Gomez-Ospina N, Bernstein JA: Clinical, cytogenetic, and molecular outcomes in a series of 66 patients with Pierre Robin sequence and literature review: 22q11.2 deletion is less common than other chromosomal anomalies. Am J Med Genet A 170A:870-880 (2016).

-Gordon CT, Tan TY, Benko S, Fitzpatrick D, Lyonnet S, Farlie PG: Long-range regulation at the SOX9 locus in development and disease. J Med Genet 46:649-656 (2009).

-Gordon CT, Attanasio C, Bhatia S, Benko S, Ansari $\mathrm{M}$, et al: Identification of novel craniofacial regulatory domains located far upstream of SOX9 and disrupted in Pierre Robin sequence. Hum Mutat 35:1011-1020 (2014).
Hanson JW, Smith DW: U-shaped palatal defect in the Robin anomalad: developmental and clinical relevance. J Pediatr 87:30-33 (1975).

-Holder-Espinasse M, Abadie V, Cormier-Daire V, Beyler C, Manach Y, et al: Pierre Robin sequence: a series of 117 consecutive cases. J Pediatr 139:588-590 (2001).

Hollanda LM, Lima CS, Cunha AF, Albuquerque DM, Vassallo J, et al: An inherited mutation leading to production of only the short isoform of GATA-1 is associated with impaired erythropoiesis. Nat Genet 38:807-812 (2006).

Izumi K, Konczal LL, Mitchell AL, Jones MC: Underlying genetic diagnosis of Pierre Robin sequence: retrospective chart review at two children's hospitals and a systematic literature review. J Pediatr 160:645-650 (2012).

Jamshidi N, Macciocca I, Dargaville PA, Thomas $\mathrm{P}$, Kilpatrick N, et al: Isolated Robin sequence associated with a balanced $\mathrm{t}(2 ; 17)$ chromosomal translocation. J Med Genet 41:e1 (2004).

Knottnerus AC, de Jong DJ, Haumann TJ, Mulder JW: Higher incidence of twins in infants with Pierre Robin sequence. Cleft Palate Craniofac J 38:284 (2001).

Li HY, Lo LJ, Chen KS, Wong KS, Chang KP: Robin sequence: review of treatment modalities for airway obstruction in 110 cases. Int J Pediatr Otorhinolaryngol 65:45-51 (2002).

Marques IL, Barbieri MA, Bettiol H: Etiopathogenesis of isolated Robin sequence. Cleft Palate Craniofac J 35:517-525 (1998).

-Matys V, Kel-Margoulis OV, Fricke E, Liebich I, Land S, et al: TRANSFAC and its module TRANSCompel: transcriptional gene regulation in eukaryotes. Nucleic Acids Res 34:D108-110 (2006).

Mehaffey MG, Newton AL, Gandhi MJ, Crossley M, Drachman JG: X-linked thrombocytopenia caused by a novel mutation of GATA-1. Blood 98:2681-2688 (2001).

Nichols KE, Crispino JD, Poncz M, White JG, Orkin SH, et al: Familial dyserythropoietic anaemia and thrombocytopenia due to an inherited mutation in GATA1. Nat Genet 24:266270 (2000).

Patel KB, Sullivan SR, Murthy AS, Marrinan E, Mulliken JB: Speech outcome after palatal repair in nonsyndromic versus syndromic Robin sequence. Plast Reconstr Surg 130:577e$584 \mathrm{e}$ (2012).

Poswillo D: Observations of fetal posture and causal mechanisms of congenital deformity of palate, mandible and limbs. J Dent Res 45: 584-596 (1966).

Printzlau A, Andersen M: Pierre Robin sequence in Denmark: a retrospective populationbased epidemiological study. Cleft Palate Craniofac J 41:47-52 (2004).

Robin P: La chute de la base de la langue consideree comme une nouvelle cause de gene dans la respiration naso-pharyngienne. Bull Acad Natl Med (Paris) 89:37-41 (1923).
Sankaran VG, Ghazvinian R, Do R, Thiru P, Vergilio JA, et al: Exome sequencing identifies GATA1 mutations resulting in DiamondBlackfan anemia. J Clin Invest 122:2439-2443 (2012).

Sheffield LJ, Reiss JA, Strohm K, Gilding M: A genetic follow-up study of 64 patients with the Pierre Robin complex. Am J Med Genet 28: 25-36 (1987).

Shprintzen RJ: Pierre Robin, micrognathia, and airway obstruction: the dependency of treatment on accurate diagnosis. Int Anesthesiol Clin 26:64-71 (1988).

Shprintzen RJ: The implications of the diagnosis of Robin sequence. Cleft Palate Craniofac J 29: 205-209 (1992).

-Simon MC, Pevny L, Wiles MV, Keller G, Costantini F, Orkin SH: Rescue of erythroid development in gene targeted GATA-1- mouse embryonic stem cells. Nat Genet 1:92-98 (1992).

Smith JLS: The Pierre Robin syndrome (glossoptosis, micrognathia, cleft palate). A review of 39 cases with emphasis on associated ocular lesions. Pediatrics 27:128-133 (1961).

Smith MC, Senders CW: Prognosis of airway obstruction and feeding difficulty in the Robin sequence. Int J Pediatr Otorhinolaryngol 70: 319-324 (2006).

Smyk M, Roeder E, Cheung SW, Szafranski P, Stankiewicz P: A de novo $1.58 \mathrm{Mb}$ deletion, including MAP2K6 and mapping $1.28 \mathrm{Mb}$ upstream to $S O X 9$, identified in a patient with Pierre Robin sequence and osteopenia with multiple fractures. Am J Med Genet A 167A:1842-1850 (2015).

Tan TY, Kilpatrick N, Farlie PG: Developmental and genetic perspectives on Pierre Robin sequence. Am J Med Genet C Semin Med Genet 163C:295-305 (2013).

Thouvenin B, Djadi-Prat J, Chalouhi C, Pierrot S, Lyonnet $S$, et al: Developmental outcome in Pierre Robin sequence: a longitudinal and prospective study of a consecutive series of severe phenotypes. Am J Med Genet A 161A:312-319 (2013).

van den Elzen AP, Semmekrot BA, Bongers EM, Huygen PL, Marres HA: Diagnosis and treatment of the Pierre Robin sequence: results of a retrospective clinical study and review of the literature. Eur J Pediatr 160:47-53 (2001).

White S, Ohnesorg T, Notini A, Roeszler K, Hewitt J, et al: Copy number variation in patients with disorders of sex development due to 46,XY gonadal dysgenesis. PLoS One 6:e17793 (2011).

Williams AJ, Williams MA, Walker CA, Bush PG: The Robin anomalad (Pierre Robin syndrome) - a follow up study. Arch Dis Child 56:663-668 (1981).

Yu C, Niakan KK, Matsushita M, Stamatoyannopoulos G, Orkin SH, Raskind WH: Xlinked thrombocytopenia with thalassemia from a mutation in the amino finger of GATA-1 affecting DNA binding rather than FOG-1 interaction. Blood 100:2040-2045 (2002). 